\title{
VINCULACIÓN EMOCIONAL Y SALUD MENTAL EN ADULTOS MAYORES INSTITUCIONALIZADOS
}

\section{Emotional Bonding and Mental Health in Institutionalized Older Adults}

\author{
Maira Alejandra GonZález-Gaviria ${ }^{1}$, \\ Diana Marcela Bedoya-Gallego²
}

Recibido: $2019-05-07$

Resumen: El envejecimiento poblacional y la institucionalización son fenómenos interrelacionados con un significativo aumento a nivel mundial. Este artículo de investigación tiene por objetivo identificar la relación que existe entre las características del vínculo establecido con el cuidador formal y el estado de salud mental experimentado por los adultos mayores institucionalizados en un hogar geriátrico de la ciudad de Medellín (Colombia). Método: investigación cualitativa de tipo estudio de caso, con uso de entrevistas en profundidad y observación participante. Resultados: en este momento vital la relación con el otro media la experiencia de salud mental, en especial el lugar que ocupa el cuidador formal, tanto en la atención de necesidades médicas como en la vinculación emocional del adulto mayor institucionalizado.

Palabras clave: salud mental, adulto mayor, vínculo emocional, instituciones asistenciales, cuidado formal.
Aceptado: 2019-08-28

Abstract: Population aging and institutionalization are interrelated phenomena with a significant increase worldwide. This research article aims to identify the relationship between the characteristics of the bond established with the formal caregiver and the state of mental health experienced by older adults institutionalized in a geriatric home in the city of Medellín (Colombia). Method: qualitative case study research using in-depth interviews and participant observation. Results: at this vital moment, the relationship with the other mediates the mental health experience, especially the place that the formal caregiver occupies, both in the care of medical needs and in the emotional connection of the institutionalized older adult..

Key Words: mental health, elder, relationship satisfaction, residential care, formal care.
Para citar este artículo en APA: González-Gaviria, D. y Bedoya-Gallego, D. (2019). Vinculación emocional y salud mental en adultos mayores institucionalizados. Revista de Psicología Universidad de Antioquia, 11(2), 99121. doi: https://doi.org/10.17533/ udea.rp.v1 $1 \mathrm{n} 2 \mathrm{a} 04$
Psicóloga. Joven investigador en la Corporación Universitaria Minuto de Dios - UNIMINUTO Seccional Antioquia-Chocó. Correo: mgonzalez25@uniminuto.edu.co; https://orcid. org/0000-0001-7128-6980

2 Psicóloga. Máster en Ciencias del Matrimonio y la Familia. Estudiante de doctorado en Psicología de la Universidad del Norte (Barranquilla - Colombia). Docente e investigadora del programa de Psicología en la Corporación Universitaria Minuto de Dios - UNIMINUTO. Seccional Antioquia-Chocó. Correo: dbedoyagall@uniminuto.edu.co; http://orcid. org/0000-0003-1061-982X 


\section{Introducción}

La acelerada Transición demográfica (TD $)^{3}$ que en diferentes regiones del mundo se está presentando, indica que el grupo poblacional de adultos mayores cada vez es más grande, debido a los cambios sociales y culturales que han transformado la calidad de vida de las personas, en especial de las mujeres, mejorando así el acceso a servicios educativos, médicos, laborales, entre otros (Dulcey-Ruiz, 2010; Flórez, Villar, Puerta y Berrocal, 2015). En Latinoamérica se efectuaron rápidamente cambios relacionados con el comportamiento poblacional; como lo expresan Flórez et al. (2015), la modernización de los países desarrollados, particularmente en lo relativo a la participación de las mujeres en el ámbito laboral, influyó en la disminución de la natalidad, la mortalidad infantil y el bajo relevo generacional que, unido a la esperanza de vida promedio de la población, trae como consecuencia el aumento en el número de personas mayores de 60 ańos. Estos cambios han supuesto grandes retos para la región en materia de investigación e intervención de los fenómenos asociados, dado que los países han tenido poco tiempo para prepararse en temas relacionados con lo social, laboral y económico; de ahí que el cuidado y su ejercicio por parte del personal capacitado sean temas pendientes en las agendas institucionales (Guerrero, Ramacciotti y Zangaro, 2019).

Según datos del último censo realizado en Colombia, el grupo poblacional de adultos mayores (personas de 60 años o más) representa aproximadamente el $11 \%$ del total de la población y la esperanza de vida al nacer pasó de 67.99 años a 76.15 años entre los años 1985-1990 a 2015-2020, por lo cual se espera que este grupo poblacional continúe aumentando (Ministerio de Salud y Protección Social, 2018).

Ya que la TD es un fenómeno complejo, desde la perspectiva crítica de la psicología social del envejecimiento (Dulcey-Ruiz, 2010) se plantea que el transcurrir de la vida hasta llegar a la adultez tardía o vejez, depende de la dinámica de factores históricos, culturales, sociales, familiares, evolutivos y psicológicos que influyen para que una persona adquiera las características propias del momento vital en el que se encuentre (Craig y Baucum, 2009; Flórez et al., 2015).

3 Aumento sustancial en la cifra de personas mayores de 60 años (Dulcey-Ruiz, 2010).

DOI: https://doi.org/10.17533/ udea.rp.v11n2a04 
Esto evidencia que los recursos sociales y las condiciones socioculturales son fundamentales para el estado y experiencia de salud mental, pues contribuyen de manera integral al mejoramiento o adquisición de habilidades que la favorecen (De Juanas, Limón y Navarro, 2013; Mebarack, Castro, Salamanca y Quintero, 2009; Molero y Pérez-Fuentes, 2011; Restrepo y Jaramillo, 2012). La relación entre estos recursos y condiciones ha sido estudiada para conocer el estado de salud, la salud mental, la calidad de vida y las características de los vínculos disponibles en la red de apoyo de los adultos mayores.

Estudios realizados con el objetivo de explorar las representaciones sociales que los adultos mayores no institucionalizados tienen de la salud mental (Martínez, Prieto y Posada, 2009; Torres, Munguía, Aranda y Salazar, 2015), determinaron que estas inciden de manera significativa en el bienestar integral porque son el resultado de la interacción de factores cognitivos, emocionales y sociales, lo cual les permite a los adultos mayores mantener relaciones funcionales con ellos mismos y con la sociedad.

Otras investigaciones en las que se ha buscado determinar la autopercepción y percepción que tienen los adultos mayores no institucionalizados de su estado general de salud encontraron que la salud mental está relacionada con la salud biológica (Zapata-Ossa, 2015), lo que conlleva a que desde la perspectiva de estos estudios se conserve una concepción de salud entendida como ausencia de enfermedad (Castańo-Vergara y Cardona-Arango, 2015; Vargas-Santillán, Arana-Gómez, García-Hernández, Ruelas-González, Melguizo-Herrera y Ruiz-Martínez, 2017), la cual privilegia, en la intervención, adquirir estilos de vida saludables para atenuar los declives propios del proceso de envejecimiento (De Juanas et al., 2013; Rosales, García y Quiñonez, 2014).

Por otro lado, estudios que se han concentrado en población institucionalizada sostienen que el apoyo social que proveen los familiares es fundamental para que los adultos mayores perciban de manera positiva la salud mental (Cardona-Arango, Estrada-Restrepo, Chavarriaga-Maya, Segura-Cardona, Ordońez-Molina y Osorio-Gómez, 2010; Molero, Pérez-Fuentes, Gázquez y Scalvo, 2011). Del mismo modo, se identifican factores como: experimentar bienestar psicológico, participar en espacios sociales (Cardona-Arias, Álvarez-Mendieta y Pastrana-Restrepo, 2014, Molina, Meléndez y Nava- 
rro, 2008), experimentar autonomía (Guerrero-Martelo, Galván y Vázquez, 2015; Molero y Pérez-Fuentes, 2011), realizar actividad física, estar en un ambiente seguro (Vitorino, Paskulin y Vianna, 2012), experimentar plenitud espiritual, cuidar de sí (Melguizo-Herrera et al., 2014), conservar y compartir recuerdos y tener una actitud positiva (Flecha, 2015). No obstante, en la búsqueda realizada para esta investigación no se encontraron reportes de estudios empíricos que mostraran la relación entre la vinculación emocional establecida con el cuidador formal y la salud mental de los adultos mayores institucionalizados.

Con respecto a las conclusiones de las investigaciones en las que se ha explorado el apoyo social durante la adultez tardía o vejez, se encuentran dos ideas centrales. La primera refiere que los vínculos o redes sociales en el transcurrir de la vida se modifican (Arias, 2009; Arias, 2013; Cardona-Arango et al., 2010; Polizzi y Arias, 2014); la segunda da cuenta de la influencia que tiene la red de apoyo primaria en la percepción de salud, en la capacidad de afrontar eventos adversos y en la satisfacción experimentada (Cuadra-Peralta, Medina y Salazar, 2016; Fernández y Stolkiner, 2013; Salgado, Noa, Matos, Cardero, Castillo, 2016; Vivaldi y Barra, 2012).

No obstante, Cardona-Arango et al. (2010) reportan que "las redes familiares se encuentran amenazadas por el descenso de la fecundidad y se espera que en el futuro otras redes sean capaces de apoyar a [los adultos mayores]" (p. 416), postura afín a la de Polizzi y Arias (2014) y Fernández y Stolkiner (2013); así pues, es importante considerar que la institucionalización es una tendencia en aumento (Flecha, 2015). Al respecto, Calzada y Ocampo (2018) plantean que la inserción laboral de los cuidadores primarios desplazó las funciones de cuidado y supervisión hacia cuidadores formales que asisten las necesidades biológicas, psicológicas, sociales, funcionales y espirituales de los adultos mayores.

Si bien en Colombia hay reportes actualizados, en el censo poblacional del 2018 no se discriminó en qué tipo de vivienda residen las personas de este grupo etario (Departamento Administrativo Nacional de Estadística [DANE], 2018); un dato del censo previo indica que los adultos mayores institucionalizados se concentran, principalmente, en hogares geriátricos privados (DANE, 
2005), definidos según la Ley 1251 de 2008 (Congreso de la República) como instituciones de atención "públicas, privadas o mixtas que cuentan con infraestructuras físicas (propias o ajenas) en donde se prestan servicios de salud o asistencia social y, en general, las dedicadas a la prestación de servicios de toda índole que beneficien al adulto mayor".

Se puede evidenciar que las investigaciones acerca de la dimensión afectiva del vínculo cuidador formal-adulto mayor en condición de institucionalización y su relación con el estado de salud mental experimentado por los adultos mayores son temas que requieren de la atención de la comunidad científica; en consecuencia, esta investigación buscó identificar la relación que existe entre las caracteristicas del vínculo establecido con el cuidador formal y el estado de salud mental experimentado por los adultos mayores institucionalizados en un hogar geriátrico de la ciudad de Medellín (Colombia).

\section{Método}

\section{Tipo y diseño de investigación}

La investigación se realizó desde un enfoque cualitativo, que enfatiza la comprensión del fenómeno a partir de los significados que los participantes atribuyen a sus experiencias (Hernández, Fernández y Baptista, 2010). Se recurrió, además, al método de estudio de caso, con el fin de lograr que la recolección, análisis y presentación de la información fuera detallada y estructurada, conservando las características particulares de los sujetos de investigación (Galeano, 2012).

\section{Participantes}

Las participantes del estudio fueron tres adultas mayores institucionalizadas en un hogar geriátrico de la ciudad de Medellín (Colombia); esta institución, de carácter privado, se encuentra ubicada en la comuna 12 del sector centro-occidente de Medellín (Colombia). Para el momento de la investigación se encontraban en calidad de institucionalización 12 personas (cuatro hombres 
y ocho mujeres) con una edad promedio de 74 años. Este hogar cuenta con un personal conformado por seis enfermeras (dos contratadas a término fijo y cuatro contratadas por prestación de servicios) que se encargan de los diferentes cuidados que requieren los residentes.

La invitación a participar en la investigación se realizó mediante selección por conveniencia (Hernández et al., 2010). Dentro de los criterios de inclusión se consideró un nivel cognitivo favorable (entendido como funcionalidad de los procesos psicológicos superiores, de acuerdo con la información suministrada en los historiales clínicos de los participantes), edad igual o superior a los 60 años y un tiempo de institucionalización igual o superior a un año.

\section{Técnicas e instrumentos}

\section{Observación participante}

Se construyó una guía en la que se consignaron las anotaciones realizadas en las visitas al hogar geriátrico durante un mes, cuya frecuencia fue de tres semanales, para un total de 14 anotaciones, cada una con un registro aproximado de dos horas de visita. La observación participante (OP) permitió comprender las características de los vínculos que se establecieron entre el adulto mayor institucionalizado y el cuidador formal, a partir de la familiarización e interacción con los participantes de la investigación.

Entrevistas en profundidad

Se construyó un protocolo que guio el curso de las entrevistas, grabadas y transcritas para favorecer el análisis de la información. En total se realizaron seis encuentros (dos por participante) de aproximadamente 60 minutos de duración cada uno. El primer encuentro se guio por un protocolo de entrevista general y el segundo por preguntas particulares que se construyeron para cada participante.

El protocolo fue validado mediante una prueba piloto con dos adultos mayores institucionalizados en hogares geriátricos diferentes. Las entrevistas permitieron interpretar qué significaba para los adultos mayores encontrarse 
en condición de institucionalización, dar cuenta del estado de salud mental ${ }^{4}$ que experimentaban y profundizar en las características de los vínculos emocionales.

\section{Procedimiento}

El análisis de la información se realizó a través de matrices categoriales divididas en componentes relativos a la OP y a las entrevistas; las unidades observacionales y las categorías se establecieron previamente con base en los objetivos específicos de la investigación, y los códigos emergieron a partir del análisis del discurso. Adicionalmente, durante el análisis de la información se consideró como categoría emergente la comprensión de aspectos del pasado de las participantes relacionados con su condición actual.

A partir de una matriz categorial general se integró la información y los resultados se construyeron a partir de la saturación del dato (Martínez-Salgado, 2012).

\section{Consideraciones éticas}

La información presentada cumple con las normativas científicas, técnicas y administrativas para la investigación en salud en Colombia (Resolución n. ${ }^{\circ}$ 0008430, 1993). Se consideró el respeto a la dignidad, los derechos y la integridad de los participantes, por lo cual se obtuvo el consentimiento informado del representante legal del hogar geriátrico para efectuar la investigación en el establecimiento que administra y acceder a los historiales clínicos de los residentes, y de cada participante para la aplicación de los instrumentos de recolección de información.

\footnotetext{
4 Se parte del concepto propuesto por Labiano (2010), desde el cual la salud mental se entiende como un sentimiento subjetivo en el que se autopercibe que el organismo desde lo biológico y psicológico funciona de manera congruente e integrada; asimismo, como un proceso dinámico y cambiante que integra las relaciones interpersonales, lo que permite que los individuos respondan al contexto en función de las exigencias de este.
}

DOI: https://doi.org/10.17533/ udea.rp.v11n2a04 


\section{Resultados}

El análisis de la información, obtenida tanto a través de la OP como de las entrevistas, permitió la identificación de 63 códigos que se relacionaron con las tres categorías de análisis propuestas: estado de salud mental experimentado, significación otorgada a la condición de institucionalización y vinculación emocional. A continuación, se describe cada una de ellas.

\section{Estado de salud mental experimentado}

La experiencia de salud mental está relacionada con la atención y cuidado que brinda el cuidador formal y con los vínculos que se establecen con otros residentes, puesto que se privilegia la relación cercana, en especial si se trata de un vínculo que proporciona apoyo y genera satisfacción, y, además, estará disponible para soportar eventos adversos que pueden llegar a presentarse en ese momento del curso de la vida, permitiendo así una ganancia bidireccional en la relación yo-otro (ver testimonio de P2 en tabla 1).

La experiencia de salud mental también tiene que ver con actividades que dependen del estilo de vida que a lo largo de los ańos los participantes han sostenido. En el proceso de institucionalización promover la participación en actividades de socialización, formación, capacitación, recreación, entre otras, transforma la experiencia de vejez, en tanto el encuentro entre el estilo de vida y las nuevas experiencias se convierte en un mecanismo adecuado para sintetizar los saberes aprendidos durante la vida. Un elemento relevante relacionado con este aspecto es el sostenimiento de la autonomía en las Actividades de la Vida Diaria (AVD) (Cardona, Estrada y Agudelo, 2006) como el autocuidado, la toma de decisiones y la interacción con el otro (ver testimonio de P1 en tabla 1).

Por consiguiente, se comprende que la salud mental en los adultos mayores institucionalizados se favorece mediante el apoyo social que brinda la persona encargada del cuidado mediante el acompañamiento en prácticas formales (basadas en la medicina convencional, por ejemplo: dar los medicamentos prescritos) o informales (basadas en la medicina alternativa, por ejemplo: ofre- 
cer infusiones, masajes), así como el apoyo brindado por otros adultos mayores institucionalizados mediante la relación establecida en espacios recreativos. Esto se manifiesta en el discurso de las participantes cuando se privilegia la experiencia de afecto en las prácticas de cuidado y autocuidado en relación con la salud física (ver testimonio de $\mathrm{P} 3$ en tabla 1 ).

\section{Tabla 1}

Estado de salud mental experimentado

\begin{tabular}{|c|c|c|}
\hline Registro & Fragmento & Código \\
\hline $\begin{array}{l}\text { Participante } 2 \text { (P2), } \\
\text { entrevista } 2 \text {, líneas } \\
313-321\end{array}$ & $\begin{array}{l}\text { Todos necesitamos afecto en la vida, si ella me ve } \\
\text { triste, lo abraza a uno como si fuera la mamá o lo } \\
\text { que sea; y: "No se ponga triste", y le da consejos } \\
\text { a uno y todo eso. (...) A mí no me visitan casi } \\
\text { y ver que nadie lo voltea a ver y nadie lo visita, } \\
\text { por ahí de vez en cuando, entonces eso en la parte } \\
\text { psíquica, mental, tiene que influir estar con una } \\
\text { persona que sea amable, querida conmigo, pues } \\
\text { es muy distinta la cosa, ¿verdad? no es solamente } \\
\text { familia, sino (...) la gente del común que sea } \\
\text { formal con uno (...) y lo consideran porque uno } \\
\text { ya está viejo y enfermo. }\end{array}$ & $\begin{array}{l}\text { Experiencia de salud men- } \\
\text { tal: relaciones sociales }\end{array}$ \\
\hline $\begin{array}{l}\text { Participante } 1(\mathrm{P} 1) \text {, } \\
\text { entrevista 1, líneas } \\
361-364\end{array}$ & $\begin{array}{l}\text { Que uno tenga buena relación con las personas, } \\
\text { que tenga alguna actividad, pongamos fuera de } \\
\text { aquí, que comparta una actividad con gente de } \\
\text { un costurero; por ejemplo, aquí en la iglesia hay } \\
\text { costurero, ¿cierto?, muchas actividades, entonces } \\
\text { eso le ayuda a uno mucho a la salud mental, com- } \\
\text { partir con las personas en alguna actividad. }\end{array}$ & $\begin{array}{l}\text { Experiencia de salud men- } \\
\text { tal: relaciones sociales }\end{array}$ \\
\hline $\begin{array}{l}\text { Participante } 3 \text { (P3) } \\
\text { entrevista } 2 \text {, líneas } \\
214-223\end{array}$ & $\begin{array}{l}\text { ¿Y cómo es eso de que ella le hace los masajes } \\
\text { con mucho fundamento? (...) Ah sí, ella es muy } \\
\text { formal y me hace mis masajes muy bien, todo } \\
\text { eso. ¿Y para usted qué significa esa atención que } \\
\text { recibe, esos masajes? Ah, una alegría, muy bueno, } \\
\text { humm. ¿Podría decirme por qué? ¡Ay!, porque a } \\
\text { mí me parece muy horrible que digan: "Ve, jle voy } \\
\text { a hacer los masajes, así! [el entrevistado utiliza un } \\
\text { tono hostil para ejemplificar una actitud durante } \\
\text { la atención], como con repelencia; me parece muy } \\
\text { horrible. }\end{array}$ & $\begin{array}{l}\text { Atención y cuidado: posi- } \\
\text { ción asumida }\end{array}$ \\
\hline
\end{tabular}

Nota. Elaboración propia. 


\section{Significación otorgada a la condición de institucionalización}

Las causas que determinaron el ingreso al hogar geriátrico inciden en las experiencias y en la percepción construida de los adultos mayores sobre su condición de vida actual (ver testimonio de P1 y P2 en tabla 2). Cuando se reside en un hogar geriátrico para solventar dificultades económicas, que al ser superadas permitirán retornar a la vida anterior, se vivencia un estado pasajero de institucionalización que puede convertirse en un factor que impide asumir la

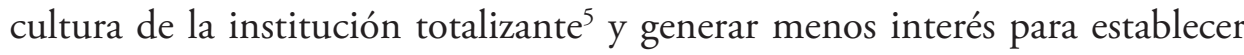
vínculos cercanos o íntimos con los cuidadores formales y otros residentes (ver testimonio de P1 en tabla 2). Por el contrario, residir en un hogar geriátrico por la búsqueda de apoyo asociada a la condición de salud implica que si un adulto mayor encuentra satisfacción en las necesidades de atención y cuidado, experimentará afectos positivos que le permiten continuar la estimulación y el desarrollo de habilidades personales y establecer relaciones sociales que le produzcan bienestar (ver anotación de OP en tabla 2).

Los procesos de interacción institucional influyen en la significación de esta condición de vida. Las normas sociales son uno de estos procesos que caracterizan las relaciones vinculares que se establecen entre los adultos mayores institucionalizados y el cuidador formal, aun cuando para el caso de este hogar geriátrico sean tácitas y laxas, , en tanto definen una rutina y un estilo de convivencia con el que se busca favorecer el bienestar de los adultos mayores. Sin embargo, no afectan el desarrollo y sostenimiento de su autonomía en la toma de decisiones, lo que implica que el rol social que estos adultos mayores han desempeńado se conserva; lo anterior, en términos de Goffman (2001), se entiende como una baja mortificación del yo (ver testimonio de P1 en tabla 2).

Esta perspectiva institucional permite comprender la pasividad que continuamente se presenció en la cotidianidad del hogar geriátrico. En el caso de dos de las participantes (P1 y P2) esta pasividad representó un proceso de aculturación (Goffman, 2001), dado que en su vida pasada fueron personas

5 Entendida como el lugar físico en el que en el que un gran número de individuos comparten la misma situación, se encuentran aislados de la sociedad por un largo período de tiempo y comparten una rutina diaria administrada formalmente (Goffman, 2001).

DOI: https://doi.org/10.17533/ udea.rp.v11n2a04 
muy activas, vinculadas a diferentes escenarios de socialización. P1 llevaba menos tiempo en el hogar geriátrico y vio en estas condiciones una exigencia de adaptación que le implicó fortalecer su autonomía. No obstante, para P2 la pasividad en las AVD del hogar representó una afectación en su experiencia de salud mental, dado que sus condiciones físicas limitaban su capacidad de producir por ella misma otros escenarios de actividad física y de socialización. En el caso de P3 pudo observarse una mayor continuidad de sus estilos de vida anteriores y los hábitos propuestos por el hogar (ver testimonio de P1 y P2 en tabla 2).

Por otro lado, un hallazgo relevante refiere que en la condición de institucionalización algunos adultos mayores, mediante los elementos que conforman su equipo de identificación, catectizan ${ }^{6}$ un lugar de la institución totalizante (Goffman, 2001), así, no solamente se apropian de su habitación, sino que también la perciben como tranquila, generadora de alegría o privacidad; esta relación con el espacio se constituye en una estrategia que generan los adultos mayores para favorecer su adaptación, en la medida en que también les ayuda a tolerar las tensiones institucionales (ver testimonio de P3 en tabla 2).

Tabla 2

Significación otorgada a la condición de institucionalización

\begin{tabular}{|l|l|l|l|}
\hline Registro & \multicolumn{1}{|c|}{ Fragmento } & $\begin{array}{l}\text { Unidad } \\
\text { observacional }\end{array}$ & Código \\
\hline P1, entrevista 1, \\
líneas 226-229 & $\begin{array}{l}\text { Lo que pasa es que tuvimos como un revés } \\
\text { de fortuna de un robo que nos hicieron, de } \\
\text { buena plata, entonces, jno es que aquí no } \\
\text { hay que pagar!, aquí hay que pagar, aquí na- } \\
\text { die es gratuito, sino que quisimos venirnos } \\
\text { un poquito (...) para acá, a ver si podíamos } \\
\text { recuperar algo de la plata que nos deben, } \\
\text { con el tiempo, a ver; ahí estamos en ese pro- } \\
\text { ceso con un abogado, ¿̇ierto? }\end{array}$ & $\begin{array}{l}\text { Hogar } \\
\text { geriátrico: } \\
\text { motivos de } \\
\text { ingreso }\end{array}$ \\
\hline
\end{tabular}

6 Desde la teoría psicoanalítica se entiende por catexis a la carga o energía psíquica que acompaña las representaciones, un objeto, un lugar, el propio cuerpo, y en consecuencia le permite a los sujetos organizar sus percepciones y comportamientos (cf. Laplanche y Pontalis, 2004). 


\begin{tabular}{|c|c|c|c|}
\hline $\begin{array}{l}\text { P2, entrevista } 1 \text {, } \\
\text { líneas } 7-15\end{array}$ & $\begin{array}{l}\text { ¿Usted hace cuánto reside acá en el hogar? } \\
\text { Voy a cumplir dos ańos el primero de } \\
\text { octubre. Bueno, ¿y usted por qué motivos } \\
\text { se traslada a vivir acá, a este lugar? Yo } \\
\text { hace mucho tiempo vivía sola, ¿verdad?, } \\
\text { entonces estaba muy enferma; tenía un } \\
\text { apartamento en un barrio de la comuna } 16 \\
\text { del sector sur-occidente de Medellín. Me } \\
\text { puse muy muy enferma, que no era capaz ni } \\
\text { de hacer una sopa; en cama, con qué cólicos } \\
\text { del estómago y todo eso. }\end{array}$ & & $\begin{array}{l}\text { Hogar } \\
\text { geriátrico: } \\
\text { motivos de } \\
\text { ingreso }\end{array}$ \\
\hline $\begin{array}{l}\mathrm{P} 1 \text {, entrevista 2, } \\
\text { líneas } \\
6-19\end{array}$ & $\begin{array}{l}\text { Uno quisiera como... como estar más libre, } \\
\text { aunque yo puedo salir todo el día si quiero; } \\
\text { sí, y quedarme donde mis hermanas, pero } \\
\text { no es como mucho la idea de estar en un } \\
\text { hogar de estos, ¿sí me entiendes?, como que } \\
\text { no me ubico total, como que no es mi am- } \\
\text { biente. }\end{array}$ & & $\begin{array}{l}\text { Hogar } \\
\text { geriátrico: } \\
\text { percepción }\end{array}$ \\
\hline $\begin{array}{l}\text { OP4, 27, junio, } \\
\text { 2017, líneas 9-10 }\end{array}$ & $\begin{array}{l}\text { Al llegar de visita al hogar geriátrico se evi- } \\
\text { dencia que en el recibidor la participante } 3 \\
\text { y W [residente] se encuentran pintando, } \\
\text { en su libro de estimulación cognitiva, una } \\
\text { mandala [esta actividad se desarrolló con } \\
\text { tranquilidad mientras P1 y P2 establecían } \\
\text { contacto, preocupándose mutuamente por } \\
\text { sus estados de salud y actividades del día } \\
\text { pendientes]. }\end{array}$ & $\begin{array}{l}\text { Residentes: } \\
\text { actividades } \\
\text { desempeñadas }\end{array}$ & $\begin{array}{l}\text { Hogar } \\
\text { geriátrico: } \\
\text { actividades de } \\
\text { estimulación }\end{array}$ \\
\hline $\begin{array}{l}\text { P1, entrevista } 1 \text {, } \\
\text { líneas } 272-277\end{array}$ & $\begin{array}{l}\text { Se asume esa responsabilidad con qué fin; } \\
\text { pues con qué fin (...) no dar como motivos } \\
\text { en el hogar de que no respeta uno las llega- } \\
\text { das, que llega tarde demasiado, no, ¿cierto? } \\
\text { Uno es consciente de que debe llegar nor- } \\
\text { mal, no llegar pasadas las horas; si yo llego } \\
\text { a las siete u ocho no hay problema, pero no } \\
\text { pasarse uno tampoco (...) por respeto. }\end{array}$ & & $\begin{array}{l}\text { Normas de } \\
\text { convivencia: } \\
\text { posición } \\
\text { asumida }\end{array}$ \\
\hline $\begin{array}{l}\text { P1, entrevista 2, } \\
\text { líneas } 41-46\end{array}$ & $\begin{array}{l}\text { Entonces yo trato de caminar, de leer y } \\
\text { hacer otra actividad, por lo menos salir, } \\
\text { ¿cierto?, yo camino mucho por acá; (...) y } \\
\text { sí: produce como más soledad (...), aunque } \\
\text { uno se hace su ambiente, pues yo veo tele- } \\
\text { visor o leo, doy mis vueltas por acá y tengo } \\
\text { una familiar también, por aquí cerquita. En } \\
\text { fin, yo trato de hacer el ambiente en el día, } \\
\text { que no esté pues totalmente encerrada. }\end{array}$ & & $\begin{array}{l}\text { Hogar } \\
\text { geriátrico: } \\
\text { actividades } \\
\text { desempeñadas }\end{array}$ \\
\hline
\end{tabular}

DOI: https://doi.org/10.17533/ udea.rp.v11n2a04 


\begin{tabular}{|l|l|l|l|}
\hline P2, entrevista 1, & $\begin{array}{l}\text { Piense en un día cotidiano de su estancia } \\
\text { acá, ¿usted qué hace? ¡Nada! Hay veces me } \\
\text { pongo a arreglar esto, porque como ellas, } \\
\text { ella me ayuda tanto a mí [F, encargada de la } \\
\text { limpieza del hogar], entonces yo también: } \\
\text { me pongo a barrer, a trapear, a sacudir, a } \\
\text { lavar; hay días que me provoca y me hago } \\
\text { el almuercito (...), como no puedo salir, } \\
\text { entonces estoy viendo televisión, a rezar un } \\
\text { poco y así muy aburrida; es lo único. Nadie } \\
\text { viene aquí, yo quisiera en este momento, } \\
\text { así me doliera todo, irme por ahí a caminar } \\
\text { unas cuadritas. }\end{array}$ & $\begin{array}{l}\text { Hogar } \\
\text { geriátrico: } \\
\text { actividades } \\
\text { desempeñadas }\end{array}$ \\
\hline P3, entrevista 2, \\
líneas 29-36 & $\begin{array}{l}\text { Me gustó mucho, mucha alegría. ¿Y por } \\
\text { qué alegría? Porque yo vivo muy contenta } \\
\text { en esta pieza, pues porque yo desde que lle- } \\
\text { gué aquí llegué a esta pieza. (...) ¿̇ay algo } \\
\text { más que implique estar en esta pieza, es de- } \\
\text { cir, más allá de que haya sido la pieza donde } \\
\text { usted haya llegado? No, a mí me gustó aquí } \\
\text { yentonces ya me dejaron aquí, entonces, ya } \\
\text { vivo muy amañada aquí. }\end{array}$ & Vivencia de \\
instituciona- \\
lización
\end{tabular}

Nota. Elaboración propia.

\section{Vinculación emocional}

El ambiente de institucionalización introduce formas específicas para la creación de vínculos; se encontró que los quebrantos de salud se convierten en el vehículo para el establecimiento de contacto y con ello la posibilidad de generar vínculos a partir de la experiencia de atención y cuidado, lo que a su vez se asocia con la percepción de que este será un recurso disponible para sobreponerse a los momentos de adversidad (ver testimonio de P3 en tabla 3). Reconocer que la condición médica media la relación permitió identificar que esta mediación cumple una función bisagra en cuanto se convierte en factor de cercanía o distanciamiento.

La forma de relación propiciada por el ambiente institucional se corresponde con las tendencias de vinculación identificadas en las participantes, de tal manera que para P1 y $\mathrm{P} 3$ su posibilidad de contar con una red de apoyo más amplia y de realizar otras actividades, incluso por fuera del hogar 
geriátrico, facilitó una mejor asimilación de este tipo de vínculo, en tanto se depende menos de él (ver testimonio de P1 y P3 en tabla 3). Situación contraria a la observada en $\mathrm{P} 2$, quien más que asistencia médica requería de la disponibilidad emocional del cuidador formal para el establecimiento de una relación constante (ver testimonio de P2 en tabla 3).

Otro aspecto asociado al contacto que se vive en el hogar geriátrico es la selectividad socioemocional (Flecha, 2015); es decir, las características físicas o de personalidad del otro determinan la posibilidad de relación (ver OP en tabla 3). Este aspecto se convierte en un factor de estrés adicional para el adulto mayor según el sentido que le otorgue a hechos como presentar un trastorno neurocognitivo, las actividades que otros residentes pueden o no desempeñar por su condición física, entre otros; por ejemplo, algunos residentes ya no pueden caminar o son asiduos fumadores (ver testimonio de P2).

Debido a que las condiciones de salud no son la única razón para requerir o buscar la atención o el cuidado, se entiende que la necesidad se relativiza en cuanto las personas tienen la expectativa que el cuidador formal, a través de sus prácticas, trascienda el asistencialismo y en esa medida tenga la capacidad o disposición para desarrollar cualidades o características que le permita ofrecer una atención más humanizada (ver testimonios de P1 y P2 en tabla 3). De esta manera, como se plantea desde la teoría del apego, se establecen afectos como la empatía y la proximidad, se genera confianza en los adultos mayores institucionalizados y se favorece el bienestar a medida que les permite interpretar que el cuidador formal, si es el caso, estará disponible para acompañarlas en otras situaciones de la vida (Bowlby, 1986; 2009). 


\section{Tabla 3}

\section{Vinculación emocional}

\begin{tabular}{|c|c|c|c|}
\hline Registro & Fragmento & $\begin{array}{c}\text { Unidad } \\
\text { observacional }\end{array}$ & Código \\
\hline $\begin{array}{l}\text { P3, entrevista 1, } \\
\text { líneas } 178-185\end{array}$ & $\begin{array}{l}\text { ¿Usted qué cree, aparte del cuidado qué más } \\
\text { le puede brindar el vínculo con las enfermeras? } \\
\text { Pero qué más, no más; uno las llama: “Ay, ve!, } \\
\text { que esto", "Vení y me hacés el favor de colocar } \\
\text { esto para no irme a caer de la cama", "Me hacés } \\
\text { el favor y me regalás el yogurt que me trajo mi } \\
\text { mamá" — que mi mamá casi siempre me trae un } \\
\text { yogurt y unas galletas-; cierto, y listo. Y, ¿para } \\
\text { usted qué significan los cuidados que le brindan? } \\
\text { Todo muy bien, para qué voy a hablar, pues, de } \\
\text { malos cuidados; no, nunca. }\end{array}$ & & $\begin{array}{l}\text { Atención } \\
\text { y cuidado: } \\
\text { personal de } \\
\text { hogar }\end{array}$ \\
\hline $\begin{array}{l}\text { P1, entrevista } 1 \text {, } \\
\text { líneas } 169-171\end{array}$ & $\begin{array}{l}\text { Y es que uno tampoco pues se apega como a } \\
\text { una persona: es que tiene que ser esta, esta y } \\
\text { esta; no, uno comparte con todas, con todas las } \\
\text { enfermeras, sea la del día o la de la noche, uno } \\
\text { comparte igual. }\end{array}$ & & $\begin{array}{l}\text { Relaciones } \\
\text { sociales: } \\
\text { personal del } \\
\text { hogar }\end{array}$ \\
\hline $\begin{array}{l}\text { P3, entrevista 1, } \\
\text { líneas } 153-154\end{array}$ & $\begin{array}{l}\text { Para mí todas son iguales, yo no tengo preferen- } \\
\text { cia porque para eso existe el pito [timbre], uno } \\
\text { las llama y ellas vienen. }\end{array}$ & & $\begin{array}{l}\text { Relaciones } \\
\text { sociales: } \\
\text { personal del } \\
\text { hogar }\end{array}$ \\
\hline $\begin{array}{l}\text { P2, entrevista 1, } \\
\text { líneas 305-308 }\end{array}$ & $\begin{array}{l}\text { Pero yo lo que necesito es una parte como con } \\
\text { más alegría, tener con quien charlar, digamos } \\
\text { que fuera otra [se refiere a la participante 1]. No, } \\
\text { se va sola y como tiene familiares por aquí, claro, } \\
\text { se va para donde su familia; yo le he dicho: "Si } \\
\text { yo tuviera con quien caminar, con quien", ¡ah!, } \\
\text { ni me dice. }\end{array}$ & & $\begin{array}{l}\text { Relaciones } \\
\text { sociales: entre } \\
\text { residentes }\end{array}$ \\
\hline $\begin{array}{l}\text { P2, entrevista } 2 \text {, } \\
\text { líneas } 33-40\end{array}$ & $\begin{array}{l}\text { ¿Usted ha tenido algún inconveniente con } \\
\text { alguno de los residentes, así como usted los } \\
\text { describe, que debían estar en un psiquiátrico? Sí, } \\
\text { es que hay unos muy groseros, está bien que están } \\
\text { enfermas y que tienen mal de Alzheimer, pues, } \\
\text { no sé, desconozco si eso cobija la grosería, que lo } \\
\text { traten a uno mal y de todo, entonces por eso es } \\
\text { que no trato con ninguna y ya. Hay algunas que } \\
\text { les han pegado y todo a las mismas enfermeras, } \\
\text { (...) le meten su juagada, y en la cara y todo. A mí } \\
\text { el otro día me insultaron dos, pero yo las regané, } \\
\text { no es el hecho de que estén enfermas para que lo } \\
\text { traten a uno así y por eso yo, para evitar todo eso, } \\
\text { me encierro acá, eso es todo. }\end{array}$ & & $\begin{array}{l}\text { Percepción de } \\
\text { la enfermedad: } \\
\text { otros residentes }\end{array}$ \\
\hline
\end{tabular}

\section{DOI: https://doi.org/10.17533/ udea.rp.v11n2a04}




\begin{tabular}{|l|l|l|l|}
\hline $\begin{array}{l}\text { OP13, 18, julio, } \\
\text { 32-37, líneas }\end{array}$ & $\begin{array}{l}\text { La participante 1 en las diferentes visitas reali- } \\
\text { zadas ha mostrado ser una persona que se } \\
\text { caracteriza por su cooperación con los demás } \\
\text { residentes, tengan dificultades evidentes para } \\
\text { moverse, o no; por ejemplo, le ha colaborado a } \\
\text { la participante 3 recibiéndole la loza y llevándola } \\
\text { a la cocina; de igual modo con la participante 2. }\end{array}$ & $\begin{array}{l}\text { Características } \\
\text { entre residentes }\end{array}$ & $\begin{array}{l}\text { de la } \\
\text { personalidad: sí } \\
\text { mismo }\end{array}$ \\
\hline $\begin{array}{l}\text { P1, entrevista 2, } \\
\text { líneas 150-160 }\end{array}$ & $\begin{array}{l}\text { ¿Cómo sería esa amistad incondicional o esa } \\
\text { atención que ellas le brindan? Una amistad, } \\
\text { pues, normal (...) que con la que esté de turno } \\
\text { uno, pues, es bien y ella con uno también; uno } \\
\text { le brinda carińo o algo que necesite (...) la soli- } \\
\text { cita con cultura, en fin, pero que uno se pegue a } \\
\text { una enfermera muy amiga, que no (...) normal, } \\
\text { la que esté en la noche o en el día, normal, pero } \\
\text { te digo: todas son bien, bien con uno. }\end{array}$ & $\begin{array}{l}\text { Relaciones } \\
\text { sociales: } \\
\text { personal de } \\
\text { hogar }\end{array}$ \\
\hline $\begin{array}{l}\text { P2, entrevista 1, } \\
\text { líneas 232-236 }\end{array}$ & $\begin{array}{l}\text { Volviendo un poco a lo que hablábamos sobre } \\
\text { F o sobre H, ¿ese vínculo a usted qué le brinda? } \\
\text { iQué me brinda!, a mí me gusta mucho que al- } \\
\text { guien se preocupe por mí, que como ser humano } \\
\text { alguien... que yo le duela a alguien, alguien se } \\
\text { interese por qué, qué me está pasando, que yo no } \\
\text { [me] sienta como un mueble: ahí lo mismo da. }\end{array}$ \\
\hline
\end{tabular}

Nota. Elaboración propia.

\section{Discusión y conclusiones}

Coincidente con las investigaciones realizadas por Martínez et al. (2009), Torres et al. (2015) y Cardona, Segura, Garzón, Segura y Cano (2016), se evidencia que los adultos mayores en condición de institucionalización al definir la noción salud mental otorgan gran importancia a los procesos cognitivos como la memoria y el pensamiento, pues ello supone la conservación de la autonomía en las AVD, lo que a su vez conlleva a que la salud mental se asocie con la capacidad de ocuparse de sí, la toma de decisiones y la interacción con el otro (Castaño-Vergara y Cardona-Arango, 2015; De Juanas, et al., 2013; Rosales, et al., 2014; Vargas-Santillán, et al., 2017; Zapata-Ossa, 2015). Llama la atención la valoración otorgada al apoyo social que brinda la nueva red establecida, considerado como aquel factor que procura mayor satisfacción y bienestar emocional, lo cual muestra que las redes sociales no solamente cam- 
bian, sino que influyen de manera significativa en el estado de salud mental experimentado de los adultos mayores institucionalizados, tal como sucede con la red de apoyo primaria (Cardona-Arango, et al., 2010; Fernández y Manrique-Abril, 2010; Salgado, et al., 2016).

Dado lo anterior, se realiza un contraste entre las ganancias que proporcionan los diferentes vínculos de la nueva red de apoyo. Por un lado, se tiene el vínculo establecido con otros residentes del hogar geriátrico; similar a lo propuesto por Flecha (2015) hay una selectividad socioemocional, esto quiere decir que si bien la formación de nuevos vínculos es un hecho innegable, el número de vínculos cercanos o íntimos se reduce, pues en el contacto con el otro se espera encontrar recursos específicos, entre otros, de orden social, emocional o intelectual, y, en caso de no estar presentes, se restringe o se impide el contacto.

Por otro lado, está el vínculo establecido con el cuidador formal, el cual se constituye por el objetivo que tienen los hogares geriátricos y por las demandas subjetivas de los adultos mayores, como la condición de salud, la disponibilidad de los vínculos de la red cercana o íntima, las características de la personalidad y el motivo de ingreso (Cardona-Arias et al., 2014, Molina et al., 2008). A través de este vínculo se busca acompañamiento por parte del cuidador formal; así, en concordancia con Alejo, Nieves y Ruiz (2016) y Vitorino et al (2012), las personas que desempeñan dicho rol proporcionan recursos externos que favorecen el sentimiento de seguridad, en cuanto brindan un cuidado humanizado, lo cual se corresponde con los resultados de la OP que permiten plantear que la atención y cuidado brindado trascienden la profesión u oficio del cuidador formal.

De igual modo, se hace evidente la diferencia en los vínculos entre residentes y entre residente y cuidador formal, pues a los cuidadores formales se les demanda cariño y se les atribuye un saber específico que debe ser desplegado de manera integral en las diferentes prácticas de cuidado (Vanegas et al., 2010). Aspecto que implica no sólo atender a las demandas en la salud física (Ramírez, Agredo, Jerez y Chapal, 2008; Zapata-Ossa, 2015), sino también a la necesidad de apoyo social y de empatía ante las demandas emocionales de los adultos mayores; hallazgo en el que también coinciden Agudelo-Suárez, 
Posada-López y Meneses-Gómez (2015), Cardona-Arango et al. (2010) y Molero et al. (2011).

En síntesis, el estado de salud mental experimentado surge de un sentir del propio individuo y depende de las vivencias experimentadas durante la vida (Rodríguez, Collazo, Álvarez, Calero, Castañeda y Gálvez, 2015). En la condición de institucionalización, el apoyo social es determinante en las percepciones que los adultos mayores construyen de su condición de vida y del estado de salud mental experimentado; en la medida en que el otro se convierte en un agente que provee recursos externos, por ejemplo, la compañía y el apoyo, se favorece la experiencia de satisfacción porque a través de dichos recursos se suplen necesidades biológicas y psicológicas.

Teniendo en cuenta la importancia de la vinculación emocional y el apoyo social en el estado de salud mental experimentado en la adultez mayor, posteriores trabajos de investigación podrán orientarse al reconocimiento de estas categorías en la formación y capacitación del recurso humano encargado de ofrecer el cuidado formal, así como su participación en instituciones públicas y privadas que acogen al adulto mayor.

Las limitaciones de esta investigación se relacionan con la falta de inclusión de residentes con condiciones médicas complejas — como la demencia senil y el Alzheimer-, lo que permitiría mayor diversidad en los hallazgos.

En resumen, se enfatiza la necesidad de tomar en cuenta aspectos tanto del curso de la vida de los adultos mayores institucionalizados como de los procesos internos de las instituciones totalizantes (hogares geriátricos), en especial el lugar que ocupa el cuidador formal. La salud mental experimentada, la significación otorgada a la condición de institucionalización y el vínculo emocional son dimensiones de análisis interrelacionadas que aportan a la comprensión de las vivencias de los adultos mayores durante la institucionalización. 


\section{Referencias}

Alejo, J., Nieves, A. y Ruiz, L. (2016). Percepciones de los adultos mayores institucionalizados en un hogar geriátrico de la ciudad de Barrancabermeja (tesis de pregrado, Pontificia Universidad Javeriana). Repositorio institucional Pontificia Universidad Javeriana. Recuperado de https://repository.javeriana.edu.co/bitstream/ handle/10554/20443/AlejoGutierrezJennifer2016.pdf?sequence $=1$

Agudelo-Suárez, A., Posada-López, A. y Meneses-Gómez, E. (2015). Salud mental en adultos mayores atendidos en la red hospitalaria pública de Medellín, Colombia. Revista Peruana de Medicina Experimental y Salud Pública, 32(4), 701-708. doi: https://doi.org/10.17843/rpmesp.2015.324.1761

Arias, C. (2009). La red de apoyo social en la vejez. Aportes para su evaluación. Revista de Psicologia da IMED, 1(1), 147-158. doi: https://doi.org/10.18256/21755027/psico-imed.v1n1p147-158

Arias, C. (2013). El apoyo social en la vejez: la familia, los amigos y la comunidad. Revista Kairós Gerontologia, 16(4), 313-329. doi: https://doi.org/10.23925/2176-901X.2013v16i4p313-329

Bowlby, J. (1986). Vinculos afectivos: formación, desarrollo y pérdida. Madrid: Ediciones Morata.

Bowlby, J. (2009). Una base segura. Buenos Aires: Paidós.

Calzada, M. y Ocampo, J. (2018). Conceptualización de cuidador institucional de persona mayor en Colombia. Revista de Salud Pública, 20(4), 511-517. doi: http://dx.doi.org/10.15446/rsap.v20n4.66544

Cardona, D., Estrada, A. y Agudelo, H. (2006). Calidad de vida y condiciones de salud de la población adulta mayor de Medellín. Biomédica, 26(2), 506-215. doi: https://doi.org/10.7705/biomedica.v26i2.1410

Cardona, D., Segura, A., Garzón, M., Segura, A. y Cano, S. (2016). Estado de salud del adulto mayor de Antioquia, Colombia. Revista Brasileira de Geriatria e Gerontologia, 19(1), 71-86. doi: https://doi.org/10.1590/1809-9823.2016.14199

Cardona-Arango, D., Estrada-Restrepo, A., Chavarriaga-Maya, L., Segura-Cardona, Á., Ordoñez-Molina, J. y Osorio-Gómez, J. (2010). Apoyo social dignificante del adulto mayor institucionalizado. Medellín, 2008. Revista de Salud Pública, 12(3), 414-424. doi: http://dx.doi.org/10.15446/rsap

Cardona-Arias, J., Álvarez-Mendieta, M. y Pastrana-Restrepo, S. (2014). Calidad de vida relacionada con la salud en adultos mayores de hogares geriátricos, Mede- 
llín, Colombia, 2012. Revista Ciencias de la Salud, 12(2), 139-155. doi: https:// doi.org/10.12804/revsalud12.2.2014.01

Castaño-Vergara, D. y Cardona-Arango, D. (2015). Percepción del estado de salud y factores asociados en adultos mayores. Revista de Salud Pública, 17(2), 171-183. doi: $10.15446 /$ rsap.v17n2.30730

Craig, G. y Baucum, D. (2009). Desarrollo psicológico. México: Pearson educación. Recuperado de https://psiqueunah.files.wordpress.com/2014/09/desarrollo-psicologico-9-ed-craig-baucum.pdf

Cuadra-Peralta, A., Medina, E., y Salazar, K. (2016). Relación de bienestar psicológico, apoyo social, estado de salud física y mental con calidad de vida en adultos mayores de la ciudad de Arica. Limite. Revista Interdisciplinaria de Filosofía y Psicología, 11(35), 56-67. Recuperado de http://www.redalyc.org/articulo. oa?id $=83646545005$

Departamento Administrativo Nacional de Estadística (DANE) (2005). Censo general 2005. República de Colombia. Población mayor. Recuperado de https://www. dane.gov.co/files/censos/presentaciones/poblacion_adulto_mayor.pdf

Departamento Administrativo Nacional de Estadística (DANE) (2018). Boletín técnico. Censo Nacional de Población y Vivienda (CNPV) 2018 (Preliminar). Recuperado de https://www.dane.gov.co/files/censo2018/informacion-tecnica/ cnpv-2018-boletin-tecnico-2da-entrega.pdf

Dulcey-Ruiz, E. (2010). Psicología social del envejecimiento y perspectiva del transcurso de vida: consideraciones críticas. Revista Colombiana de Psicología, 19(2), 207-224. Recuperado de http://www.scielo.org.co/pdf/rcps/v19n2/v19n2a05. pdf

Fernández, R. y Manrique-Abril, F. (2010). Rol de la enfermería en el apoyo social de adulto mayor. Enfermería Global, 19, 1-9. doi: https://doi.org/10.6018/eglobal.9.2.107281

Fernández, T. y Stolkiner, A. (2013). Las prácticas de cuidado de la salud de las personas adultas mayores institucionalizadas. Anuario de Investigaciones, 20, 303310. Recuperado de http://www.redalyc.org/articulo.oa?id=369139949069

Flecha, A. (2015). Bienestar psicológico subjetivo y personas mayores residentes. Pedagogía social. Revista Interuniversitaria, 25, 319-341. doi: https://doi. org/10.7179/PSRI_2015.25.14

Flórez, C., Villar, L., Puerta N. y Berrocal L. (2015). El proceso de envejecimiento de la población en Colombia: 1985-2050. En Fedesarrollo y Fundación Saldarria- 
ga Concha, Misión Colombia envejece: cifras, retos y recomendaciones (pp. 1-79). Bogotá: Fundación Saldarriaga Concha.

Galeano, M. (2012). Estrategias de investigación social cualitativa: El giro en la mirada. Medellín: La Carreta Editores

Goffman, E. (2001). Internados. Ensayos sobre la situación social de los enfermos mentales. Buenos Aires: Amorrortu Editores. Recuperado de https://sociologiaycultura.files.wordpress.com/2014/02/goffmaninternados.pdf

Guerrero, G., Ramacciotti, K. y Zangaro, M. (2019). Los derroteros del cuidado. Bernal: Universidad Nacional de Quilmes.

Guerrero-Martelo, M., Galván, G. y Vázquez, F. (2015). Relación entre autoestima y autonomía funcional en función del apoyo social en adultos institucionalizados de la tercera edad. Psicogente, 18(34), 303-310. doi: http://dx.doi. org/10.17081/psico.18.34.506

Hernández, R., Fernández, C. y Baptista, P. (2010). Metodología de la investigación. México D.F.: McGraw-Hill.

Juanas de, Á., Limón, M. y Navarro, E. (2013). Análisis del bienestar psicológico, estado de salud percibido y calidad de vida en personas adultas mayores. Pedagogía Social. Revista Interuniversitaria, (22), 153-168. doi: 10.7179/PSRI_2013.22.02

Labiano, M. (2010). Introducción a la psicología de la salud. En L. Oblitas (Coord.), Psicología de la salud y calidad de vida (pp. 3-21). México D. F.: CengageLearning Editores.

Laplanche, J. y Pontalis, J. (2004). Diccionario de psicoanálisis. Buenos Aires: Paidós. Recuperado de https://teoriaspsicologicas2.files.wordpress.com/2013/02/ diccionario-laplanche-pontalis.pdf

Ley n. ${ }^{\circ}$ 1251, por la cual se dictan normas tendientes a procurar la protección, promoción y defensa de los derechos de los adultos mayores. Publicada por el Congreso de la República, del 27 de noviembre de 2008. Recuperado de https://www. icbf.gov.co/cargues/avance/docs/ley_1251_2008.htm

Martínez, A., Prieto, A. y Posada, M. (2009). Una mirada a la salud mental positiva en adultos mayores: grupos focales. Revista Nacional de Investigaciones-Memorias, 5(12), 80-90. Recuperado de https://revistas.ucc.edu.co/index.php/me/ article/view/217/216

Martínez-Salgado, C. (2012). El muestreo en investigación cualitativa. Principios básicos y algunas controversias. Ciência \& Saúde Coletiva, 17(3), 613-619. doi: https://doi.org/10.1590/S1413-81232012000300006 
Mebarack, M., Castro, A., Salamanca, M. y Quintero, M. (2009). Salud mental: un abordaje desde la perspectiva de la psicología de la salud. Psicología desde el Caribe, (23), 83-112. Recuperado de http://www.scielo.org.co/pdf/psdc/n23/ n23a06.pdf

Melguizo-Herrera, E., Ayala-Medrano, S., Grau-Coneo, M., Merchán-Duitama, A., Montes-Hernández, T., Payares-Martínez., C. y Reyes-Villadiego, T. (2014). Calidad de vida de adultos mayores en centros de protección social en Cartagena (Colombia). Aquichan, 14 (3), 537-548. doi: http://dx.doi.org/10.5294/ aqui.2014.14.4.8

Ministerio de Salud y Protección Social. Oficina de la Promoción Social. (2018). Sala situacional de la población adulta mayor. Recuperado de https://www.minsalud.gov.co/sites/rid/Lists/BibliotecaDigital/RIDE/DE/PS/sala-situacion-envejecimiento-2018.pdf

Molero, M. y Pérez-Fuentes, M. (2011). Salud y calidad de vida en adultos mayores institucionalizados. Revista INFAD de Psicología, 4(1), 249-258. Recuperado de http://infad.eu/RevistaINFAD/2011/n1/volumen4/INFAD_010423_249-258.pdf

Molero, M., Pérez-Fuentes, M., Gázquez, J. y Scalvo, E. (2011). Apoyo familiar en mayores institucionalizados. European Journal of Investigation in Health, Psychology and Education, 1(1), 31-43. doi: https://doi.org/10.30552/ejihpe.v1i1.14

Molina, C., Meléndez, J. y Navarro, E. (2008). Bienestar y calidad de vida en ancianos institucionalizados y no institucionalizados. Anales de psicología, 24(2) 312-319. Recuperado de http://www.um.es/analesps/v24/v24_2/14-24_2.pdf

Polizzi, L. y Arias, C. (2014). Los vínculos que brindan mayor satisfacción en la red de apoyo social de los adultos mayores. Pensando Psicología, 10(17), 61-70. doi: https://doi.org/10.16925/pe.v10i17.784

Ramírez, R., Agredo, R., Jerez, A. y Chapal, L. (2008). Calidad de vida y condiciones de salud en adultos mayores no institucionalizados en Cali, Colombia. Revista de Salud Pública, 10(4), 529-536. Recuperado de http://www.scielo.org.co/scielo.php?pid=S0124-00642008000400003\&script=sci_abstract\&tlng=es

Restrepo, D. y Jaramillo, J. (2012). Concepciones de salud mental en el campo de la salud pública. Revista Facultad Nacional de Salud Pública, 30(2), 202-211. Recuperado de http://www.scielo.org.co/pdf/rfnsp/v30n2/v30n2a09.pdf

Resolución n. ${ }^{\circ} 0008430$ por la cual se establecen las normas científicas, técnicas y administrativas para la investigación en salud. Publicada por el Ministerio de Salud, del 04 de octubre de 1993. Recuperado de https://www.min- 
salud.gov.co/sites/rid/Lists/BibliotecaDigital/RIDE/DE/DIJ/RESOLUCION-8430-DE-1993.PDF

Rodríguez, A., Collazo, M., Álvarez, L., Calero, J., Castañeda, I y Gálvez, A. (2015). Necesidades de atención en salud percibidas por adultos mayores cubanos. Revista Cubana de Salud Pública, 41(3), 401-412. Recuperado de http://scielo.sld. cu/pdf/rcsp/v41n3/spu02315.pdf

Rosales, R., García, R. y Quiñones, E. (2014). Estilo de vida y autopercepción sobre salud mental en el adulto mayor. MEDISAN, 18(1), 61-67. Recuperado de http:// scielo.sld.cu/scielo.php?script=sci_arttextypid=S1029-30192014000100009

Salgado, M., Noa, M., Matos, D., Cardero, D., y Castillo, Y. (2016). Influencia de algunos factores biopsicosociales en el bienestar subjetivo de adultos mayores institucionalizados. MEDISAN, 20(10), 4045-4051. Recuperado de http:// www.redalyc.org/articulo.oa?id=368447678001

Torres, T., Munguía, J., Aranda C. y Salazar J. (2015). Representaciones sociales de la salud mental y enfermedad mental de población adulta de Guadalajara, México. Revista CES Psicología, 8(1), 63-76. Recuperado de http://www.redalyc.org/ articulo.oa?id $=423539425005$

Vanegas, B., Vargas, E., Abella, L., Alayón, M., Bonza, L., Chaparro, Y., Espinoza, J., Forero, M. y Gutiérrez, C. (2010). Roles que desempeñan profesionales de enfermería en instituciones geriátricas de Bogotá. Revista Colombiana de Enfermería, 5(1), 81-92. doi: https://doi.org/10.18270/rce.v5i5.1427

Vargas-Santillán, M., Arana-Gómez, B., García-Hernández, M., Ruelas-González, M., Melguizo-Herrera, E., y Ruiz-Martínez, A. (2017). Significado de salud: la vivencia del adulto mayor. Aquichan, 17(2), 171-182. doi: https://doi. org/10.5294/aqui.2017.17.2.6

Vitorino, L., Paskulin, L. y Vianna. L. (2012). Calidad de vida de mayores en institución de larga permanencia. Rev. Latino-Am. Enfermagem, 20(6), 1186-1195. doi: https://doi.org/10.1590/S0104-11692012000600022

Vivaldi, F. y Barra, E. (2012). Bienestar psicológico, apoyo social percibido y percepción de salud en adultos mayores. Terapia Psicológica, 30(2), 23-29. doi: http:// dx.doi.org/10.4067/S0718-48082012000200002

Zapata-Ossa, H. (2015). Autopercepción de salud en adultos mayores y desenlaces en salud física, mental y síndromes geriátricos, en Santiago de Cali. Revista de Salud Pública, 14(4), 589-602. doi: http://dx.doi.org/10.15446/rsap.v17n4.36944 
